\title{
Lets go Surfing now, everybody's learning how; attentional strategies on expert and novice surfing performance under both practice and competition conditions
}

\author{
Lawrence, Gavin P; Virian, Jana; Oliver, Samuel J; Gottwald, Victoria M
}

\section{European Journal of Sport Science}

DOI:

10.1080/17461391.2019.1626489

Published: 01/01/2020

Peer reviewed version

Cyswllt i'r cyhoeddiad / Link to publication

Dyfyniad o'r fersiwn a gyhoeddwyd / Citation for published version (APA):

Lawrence, G. P., Virian, J., Oliver, S. J., \& Gottwald, V. M. (2020). Lets go Surfing now, everybody's learning how; attentional strategies on expert and novice surfing performance under both practice and competition conditions. European Journal of Sport Science, 20(2), 229-239. https://doi.org/10.1080/17461391.2019.1626489

\footnotetext{
Hawliau Cyffredinol / General rights

Copyright and moral rights for the publications made accessible in the public portal are retained by the authors and/or other copyright owners and it is a condition of accessing publications that users recognise and abide by the legal requirements associated with these rights.

- Users may download and print one copy of any publication from the public portal for the purpose of private study or research.

- You may not further distribute the material or use it for any profit-making activity or commercial gain

- You may freely distribute the URL identifying the publication in the public portal ?
}

Take down policy

If you believe that this document breaches copyright please contact us providing details, and we will remove access to the work immediately and investigate your claim. 
Publisher: Taylor \& Francis \& European College of Sport Science

Journal: European Journal of Sport Science

DOI: $10.1080 / 17461391.2019 .1626489$

(A) Check for updates

Lets go Surfing now, everybody's learning how; attentional strategies on expert and novice surfing performance under both practice and competition conditions.

Gavin P. Lawrence ${ }^{\mathrm{a}}$

Jana Virian ${ }^{\mathrm{a}}$

Samuel J. Oliver ${ }^{\mathrm{a}}$

Victoria M. Gottwald ${ }^{\mathrm{a}}$

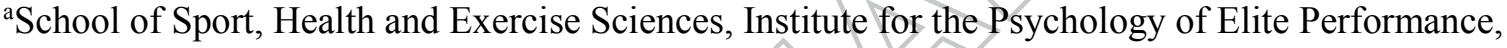

Bangor University.

Corresponding Author: Dr Gavin P Lawrence

School of Sport, Health and Exercise Sciences,

Institute for the Psychology of Elite Performance,

Bangor University,

George Building,

Holyhead Road,

Bangor,

Gwynedd.

LL57 2PZ

E-mail: g.p.lawrence@bangor.ac.uk

Tel: $\quad+44(0) 1248388283$

Acknowledgement

This work was supported by Knowledge Economy Skills Scholarships (KESS II; grant ID number:

80815) in cooperation with Surf Snowdonia. KESS II is a major European Convergence programme led by Bangor University on behalf of the HE sector in Wales.

- Manuscript word count: 5282 


\begin{abstract}
Research suggests an external focus of attention (EFOA) promotes effective performance and learning compared to an internal focus of attention (IFOA), with explanations proposed by the constrained action hypothesis (CAH) and OPTIMAL theory of motor learning. Specifically, it is proposed that adopting an EFOA prevents the constraining of normally automatic control processes and subsequent performance decrements (typically observed when individuals adopt an IFOA) by simultaneously reducing self-focus strategies and enhancing goal-action coupling. The present study attempted to fill this research lacuna by examining the CAH and OPTIMAL theory in a complex, ecologically valid, motor task under both low and high-pressured conditions. In two separate experiments, novice $(n=23)$ and advanced $(n=21)$ surfers completed a surfing task in an artificial Wavegarden under either EFOA or IFOA instructions. Experimental phases included a pre-test, acquisition and competition transfer. Results revealed that advanced and novice surfing performance benefited from adopting an EFOA in both normal and competitive situations. These findings are discussed in terms of the theoretical implications for the CAH and OPTIMAL theory, in addition to the anxiety literature. Reference to applied implications when instructing and performing within ecologically valid surfing settings are also presented.
\end{abstract}

Keywords: Coaching, Performance, Stress, Expertise 


\section{Let's go Surfing now, everybody's learning how: attentional strategies on expert and novice}

surfing performance under both practice and competition conditions.

Growing interest in both recreational and competitive surfing over the last decade has culminated in the sport being officially approved as an Olympic sport ahead of Tokyo 2020 (International Surfing Association, 2016). Consequently, the relative importance of effective coaching with regards to instructions provided in both learning and pressured environments is ever growing. More specifically, the use of scientific, evidence based instructions to enhance learning has the potential to both elevate surfing as a 'medal' sport as well as increase participation figures: something which has extensive sporting as well as health and socio-economic impact. Instructional delivery is of particular importance for the learning and refinement of complex skills such as surfing, in which the control of many degrees of freedom and coordination of several movement sequences are crucial (Wulf, Höß \& Prinz, 1998).

Learning to surf, and acquiring new or refining existing manoeuvres typically involves instructions from a coach that can often direct a performer's attention to aspects of their own body position (Wulf \& Prinz, 2001). However, there is now robust evidence (see review Wulf, 2013) to suggest that focusing on body centred information (i.e., adopting an internal focus of attention: IFOA) can paradoxically be detrimental to performance when compared with focusing attention towards the effects of one's movements (i.e., an external focus of attention: EFOA). An explanation for this phenomenon has been proposed within the Constrained Action Hypothesis (CAH) (Wulf, McNevin, \& Shea, 2001). Specifically, adopting an EFOA is purported to prevent the constraining of normally automatic control processes and subsequent performance decrements typically observed when individuals adopt an IFOA (Wulf et al., 1998; 2001; Wulf \& McNevin, 2003). This is likely achieved from enhanced 'goal-action coupling' and reduced self-focus when adopting an EFOA (Wulf \& Lewthwaite, 2016). This notion forms part of Wulf and Lewthwaite's (2016) OPTIMAL (Optimizing Performance through Intrinsic Motivation and Attention for Learning) theory of motor learning. OPTIMAL theory proposes that learning consists of a complex interaction between motivational factors (associated with enhancements in both autonomy and performance expectations) and 
attentional factors regarding particular movement related focus cues. Specifically, autonomy and enhanced expectations together with an external focus are all proposed to enhance goal-action coupling which lead to a reduced self-focus and an increased task focus. Consistent with previous literature, OPTIMAL theory proposes that a shift away from adopting a self-focus and towards a goal focus leads to increased task performance, increased motor learning, and subsequently, further enhances performance expectations: one of the primary factors proposed in the theory for the increased goal-action coupling, thus creating a cycle of enhanced learning. Furthermore, Wulf and Lewthwaite make a number of interesting, if speculative, predictions concerning the mechanisms for both sensorimotor and motivational functions on goal-action coupling and performance expectations. Predictions are linked to past literature and include increased potentiation (i.e., enhanced functional connectivity through goal-action coupling) following increased dopamine responses, enhanced selfefficacy, competence, and sense of agency, together with increased automaticity paired with reduced self-reflection or concerns over muscle and joint co-ordination.

With specific reference to the attention aspect of OPTIMAL theory, the learning and performance benefits associated with adopting an EFOA have also been explained by reduced cognitive demands and thus, increased attentional capacity (Totsika \& Wulf, 2003; Wulf et al., 2001). Similarly, increased cognitive demands have been proposed to be responsible for anxiety associated negative effects on performance. Attentional Control Theory (Eysenck, Derakshan, Santos \& Calvo, 2007) suggests that under anxious conditions, attention is typically shifted away from goal-directed systems towards threat-related stimuli e.g., worry. This shift can increase the overall cognitive demands of the task situation, often resulting in a performer exceeding their attentional capacity and subsequently decreasing their performance (Mullen, Hardy, \& Tattersall, 2005). An alternative line of research, namely Conscious Processing Hypothesis (Masters, 1992) or similarly, Reinvestment Theory (Masters \& Maxwell, 2008), propose that performers are not 'distracted' under anxiety, but rather adopt a debilitative 'self-focus' (see also Baumeister, 1984). This self-focus results in participants reinvesting explicit, movement-centred information in an attempt to consciously control movements. This, in turn, disrupts performance by interfering with normally automatic response programming and control. The similarity in the proposed self-focus mechanisms underlying the 
detrimental effects of both reinvestment under anxious performance and constrained actions under an IFOA are certainly evident. Indeed, the reduced performance and learning effects associated with self-focus are a central component of the OPTIMAL theory. With the authors of that theory describing the potential impact of adopting an IFOA (self-focus) as encouraging a series of 'microchoking episodes' (Wulf \& Lewthwaite, 2010).

Previous laboratory based studies examining focus of attention in conjunction with anxiety, provide evidence for inhibition of the above processes via an EFOA. For example, Bell and Hardy (2009) investigated the effects of adopting an EFOA on pressured performance and hypothesized that the benefits were due to mechanisms within attentional control theory i.e., yia increased automaticity and reduced cognitive demands. A study by Lawrence, Gottwald, Khan and Kramer (2012), showed that participants adopting an EFOA were prevented from developing a bank of movement-centred explicit information during learning. These findings were interpreted in line with self-focus mechanisms whereby adopting an EFOA resulted in a reduction in the type of information that could be reinvested to disrupt performance when participants were anxious. This facilitative nature of an EFOA during anxious performance has typically been investigated in closed skills such as golf putting and has simultaneously never been inyestigated in advanced and novice populations.

Considering the complex and uncontrollable nature of surfing, it is not surprising that research has neglected to investigate skill acquisition strategies on surfing performance per se. The aim of the present study was to examine whether the proposed benefits associated with adopting an EFOA could be extended to the performance of a complex whole body movement task undertaken within both control and pressure conditions.

\section{Experiment 1}

Experiment 1 investigated an internal versus external focus of attention in surfing. This comparison was investigated in advanced surfers under both low and high-pressured situations in a Wavegarden creating artificial and consistent waves. This enabled the researchers to examine the effects of attentional strategies on surfing performance under pressure in an ecologically valid, but controlled setting. Examining the benefits of directing the performers' attention to movement effects 
as opposed to body movements contributes to a better understanding of attentional strategies as a potential coping mechanism under pressurised surfing performance. In line with the proposals of theories containing distraction (Beilock \& Carr, 2001; Eysenck \& Calvo, 1992; Eysenck et al., 2007; Mullen et al., 2005) and self-focus (Baumeister, 1984; Masters, 1992; Masters \& Maxwell, 2008; Wulf \& Lewthwaite, 2016; Wulf et al., 2001), it was hypothesised that adopting an EFOA would result in enhanced performance compared to an IFOA in both low and high-pressured conditions. Since previous literature has criticised the absence of manipulation checks within attentional focus designs (see Castaneda \& Gray, 2007; Collins, Carson \& Toner, 2016), a manipulation check was incorporated into the study to ensure participants were adopting the appropriate focus of attention.

\section{Methodology}

\section{Participants}

Forty-four participants volunteered to participate in the experiment and were naïve to research hypotheses. Participants were advanced surfers ( $n=21,18$ men and 3 women; mean 24.9, $S D=3.5$ years; height mean $178.0, S D=9.0 \mathrm{~cm}$; body mass mean $74.5, S D=8.8 \mathrm{~kg}$ ) and participated in the sport on average greater than three times per week with two-thirds being qualified International Surfing Association (ASI) Level 1 instructors. All participants received instructions about the experimental procedures and provided written informed consent prior to the investigation. All experimental protocols were submitted to and approved by the ethics committee of the School of Sport, Health and Exercise Sciences, Bangor University for research involving human participants.

Task

The research was conducted at the world's first commercial inland wave lagoon; Adventure Parc Snowdonia, Conwy, North Wales. A foil, which resembles a snowplough, is mechanically dragged under water creating a consistent, travelling wave over a distance of $300 \mathrm{~m}$. The task requirement for this experiment was to surf a $1.5 \mathrm{~m}$ high peeling wave over a distance of $150 \mathrm{~m}$ whilst performing instructed complex surfing manoeuvres. Each trial consisted of a single wave, with each wave lasting circa 40 seconds. Participants alternated between surfing waves on their forehand (i.e., facing the wave while surfing) and backhand (i.e., surfing with their back to the wave). Participants were given the opportunity to surf the wave lagoon for one hour for familiarisation purposes (on a 
separate day prior to the investigation). All trials were video recorded for later performance analysis, with surfing performance determined from video analysis by an experienced surfing judge (ISA qualified with 6 years of experience). The judge was blind to experimental hypotheses and focus conditions. Participants were judged according to the World Surf League (WSL) criteria, used in professional competitions with scores ranging from 1.0 to 10.0 . That is, surfers were judged by the complexity of manoeuvres performed at the most critical part of the wave with speed, power and flow. Participants were informed that their performance was being judged in line with the WSL criteria and were instructed to approach surfing the wave as they would in a competition i.e., to surf the wave in order to gain maximum points by performing manoeuvres at the most critical part of the wave with speed, power and flow. Participants were then free to choose how to surf the wave and what manoeuvers to perform. For consistency across trials, participants were asked to perform the same surfing 'routine' (i.e., to attempt the same manoeuvers) on each wave surfed'.

\section{Procedure}

Participants were informed that the purpose of the experiment was to investigate the effects of different types of instruction on surfing performance. Experimental procedures including performance measures were explained. Three experimental phases were included; pre-test, acquisition and transfer. After a pre-test, in which all participants had the opportunity to practice the task for two trials without instructions, participants were randomly assigned to either an EFOA or IFOA group. This was followed by an acquisition phase consisting of eight trials with scripted attentional focus reminders prior to each trial. The transfer phase consisted of a single trial, in which participants surfed their preferred side (forehand or backhand) under pressured conditions. No attentional instructions were provided during transfer. The rest period between trials was 4.5 mins. During acquisition, this time was used to give participants the attentional focus instructions.

\footnotetext{
${ }^{1}$ As expected, each surfer chose different routines. The level of difficult of those chosen manoeuvers naturally differed, likely because of participants' adopting different strategies during competition. However, non-significant group differences between performance scores at pre-test (see Figure 1) indicate that the difficultly of the routines chosen and how they were performed (in the IFOA and EFOA experimental groups) were comparable. This is likely due to the combination of applying an 'expertise' criteria when obtaining the sample for the experiment and through randomly allocating participants to IFOA and EFOA groups.
} 
The IFOA and EFOA instructions are shown in table 1. Whilst the instructions in the IFOA and EXFOA groups directed attention towards the body or the movement effect respectively, they were developed in order to ensure the groups were provided comparable information (in terms of content and wording) and that instructions were directed towards specific aspects of surfing. Furthermore, in both groups, participants received generic and consistent instructions (i.e., instructions were not altered for performance level or choice of routine).

Following acquisition, participants were transferred to a pressure condition, where two types of manipulations were used: monitoring apprehension and outcome-based performance pressure (Beilock, Carr, MacMahon, \& Starkes, 2002; DeCaro, Thomas, Albert, \& Beilock, 2011 ).

Specifically, monitoring and evaluation apprehension were manipulated by informing participants that their transfer trial would be video recorded. Outcome-based performance pressure was induced by informing participants that their transfer surfing performance video would be analysed by expert coaches from Surfing Great Britain. These coaches would then categorise participants into high and low performing surfing groups before subsequently publishing this information and videos on Surf Snowdonia's website and social media (i.e., Facebook, Instagram) pages.

In line with previous research (Lawrence, Cassell, Beattie, et al., 2014), after acquisition and transfer phases, participants completed a post manipulation questionnaire to assess the extent to which they adopted the respective attentional focus instructions. Specifically, participants were asked to rate their adopted respective attentional foci (whilst surfing) along a 10-point Likert scale. The scale assessed the extent to which they adopted a focus on their movements or any part of their body (e.g., legs, torso, arms, hands or head) to a focus on their board, the wave, or other markers (e.g., fence, noise, wristband). A strong movement-centred focus was reflected with a score closer to 1 and a strong movement-effect focus was reflected in a score closer to 10 .

Dependent Measures and Analyses

As per Lawrence et al., (2012, 2014), Robazza, Bortoli and Nougier (2000), and Wilson, Wood and Vine (2009) the Mental Readiness Form-3 (Krane, 1994) was used to assess the effect of the pressure manipulation immediately before the three experimental phases. The MRF-3 contains three 11-point Likert scales with anchors from 'not worried' (1) to 'worried' (11) (i.e., cognitive 
anxiety), 'not tense' to 'tense' (i.e., somatic anxiety) and 'confident' to 'not confident' (i.e., selfconfidence). For the purpose of the current experiment, only the cognitive anxiety data were analysed. The rationale for this is that perceived pressure to perform is linked to the anxiety levels specific to that performance (e.g., state anxiety) (Gucciardi, Longbottom, Jackson, \& Dimmock, 2010).

Primary analyses measured the effect of attentional focus on surfing performance and anxiety during the experimental phases. Specifically, in order to examine the experimentally hypothesized performance effects, separate independent samples t-tests were conducted to compare the means between the EFOA and IFOA groups at the pre-test, acquisition, and transfer experimental phases. Similar analyses were conducted on the MRF-3 group means of the self-reported cognitiye anxiety (worry) scale data. In addition, group differences between the changes in performance from the low and high pressure conditions were investigated by first calculating separate change scores between pre-test and transfer and between acquisition and transfer for the IFOA and EFOA groups. Means of these change score data were then compared between groups by performing separate independent samples t-tests.

\section{Results}

FOA Manipulation Check

Comparison of the manipulation check data of EFOA and IFOA groups revealed that participants in the EFOA group reported significantly higher values (mean $=6.72, S D=1.85$; values more representatiye of a movement-effect focus) compared to those in the IFOA group (mean $=2.5$, $S D=0.85$; yalues more representative of a movement-centred focus) $\left(\mathrm{t}_{(19)}=6.63, p<.001\right)$.

\section{Performance}

Performance data are presented in Figure 1A. Analyses revealed that the groups were not significantly different at pre-test $\left(\right.$ EFOA mean $=1.52, S D=0.84 ;$ IFOA mean $=2.05, S D=0.52 ; \mathrm{t}_{(19)}$ $=-1.70, p=0.105)$. However, at both acquisition $($ EFOA mean $=2.88, S D=1.29$; IFOA mean $=$ $\left.1.63, S D=1.13 ; \mathrm{t}_{(19)}=2.32, p<0.05\right)$ and transfer $($ EFOA mean $=2.63, S D=1.09$; IFOA mean $=$ $\left.1.65, S D=1.02 ; \mathrm{t}_{(19)}=2.12, p<0.05\right)$, the EFOA group performed significantly better than the IFOA group. Performance change from pre-test to transfer was significantly different between the groups 
$\left(\mathrm{t}_{(19)}=2.97, p<0.05\right)$ with performance increasing by 1.1 scores in EFOA and decreasing .40 scores in the IFOA ${ }^{2}$. Performance change from acquisition to transfer was not significantly different between the two groups $\left(t_{(19)}=-.45, p=.66\right)$.

Anxiety

Anxiety data are shown in Figure 1B. Analyses revealed that self-reported anxiety levels did not significantly differ between groups at either pre-test (EFOA mean $=4.44, S D=2.24$; IFOA mean $\left.=5.66, S D=1.80 ; \mathrm{t}_{(19)}=-1.27, p=0.221\right)$, acquisition $($ EFOA mean $=4.55, S D=2.24$, IFOA mean $=$ $\left.5.77, S D=1.64 ; \mathrm{t}_{(19)}=0.214, p=0.833\right)$, or transfer $(\mathrm{EFOA}$ mean $=5.11, S D=2,14$, IFOA mean $=$ $\left.4.88, S D=2.26 ; \mathrm{t}_{(19)}=0.21, p=0.833\right)$.

\section{Discussion}

The rationale behind Experiment 1 was to investigate focus of attention in a complex, wholebody surfing task under both high and low pressure in a sample of experts. Findings provide further support for the CAH (Wulf et al., 2001) and more recently, OPTIMAL theory of motor learning (Wulf \& Lewthwaite, 2016). That is, performance was greater when participants adopted an EFOA. Anxiety levels were not significantly different between the EFOA or IFOA groups at either the pre-test, acquisition, or transfer. The method of pressure manipulation in our transfer test has been successfully used in previous literature (Allsop, Lawrence, Khan, \& Gray, 2017; Cassell, Beattie, \& Lawrence, 2017; Lawrence et al., 2012; 2014) and the values reported are similar to those of previous literature adopting the same or similar measures (Allsop et al., 2017; Oudejans \& Pijpers, 2010). However, the levels of anxiety reported at both pre-test (overall mean $=5.05, S D=2.07$ ) and acquisition (overall mean $=5.16, S D=2.00$ ) were higher than one might have expected (for reasons of parity, we offer detailed proposals for this in the general discussion section). However, despite non-

2 The use of a single judge means there is no measure of intra- or inter-judge relatability. However, participants were judged according to the consistent and recognised World Surf League (WSL) criteria, and scored by an experienced surfing judge (ISA qualified with 6 years of experience) who was blind to experimental hypotheses and focus conditions. The SEm's of the mean performance scores for the EFOA and IFOA at pre-test, acquisition, and transfer are $0.25,0.38,0.33$ and $0.16,0.35,0.32$, respectively. Thus, we are $95 \%$ confident that the population performance means of similar ability surfers adopting an EFOA would fall between 1.02 and 2.01 at pre-test, 2.11 and 3.63 at acquisition, and 1.98 and 3.28 at transfer. For those adopting an IFOA, the same confidence intervals would result in the population performance means falling between 1.72 and 2.37 for pre-test, 0.93 and 2.34 at acquisition, and 1.01 and 2.28 at transfer. 
significant group differences in anxiety levels at all experimental phases the EFOA group did perform significantly better at both the control (acquisition) and pressure manipulation (transfer) experimental phases: explanations for these findings are presented in the general discussion section. Since previous literature has identified some potential expert-novice differences in terms of performance effects in both the stress and performance and the attentional focus literature (Beilock \& Carr, 2001; Beilock et al., 2002; Ford, Hodges \& Williams, 2005) the purpose of experiment 2 was to confirm our findings in a sample of novice surfers.

\section{Experiment 2}

\section{Introduction}

The notion of 'choking under pressure' is typically associated with 'expert' performance (see Hill, Hanton, Matthews \& Fleming, 2008). Being that the very nature of 'choking' entails performance decrements 'relative to the norm' (Beumeister, 1984; Worthy, Markman \& Maddox, 2009), it makes sense that the effects of anxiety are more pronounced when performance is habitually high.

Furthermore, the aforementioned mechanisms accounting for anxious performance suggest that it is the proceduralised nature of motor skills that is broken down under anxiety (Masters, 1992). Thus, skills that are not yet proceduralised are less likely to be affected. However, there is evidence to suggest that adopting an EFOA may still offer some performance benefits to novices performing in pressurised conditions (see Lawrence et al., 2012; Mullen, Faull, Jones \& Kingston, 2012). The rationale behind Experiment 2 was therefore to determine whether the findings of Experiment 1 would be replicated in novice performers.

\section{Methods}

\section{Participants}

Participants in Experiment 2 were novice surfers $(n=23 ; 14$ men and 9 women; age mean 24.2, $S D=3.5$ years; height mean $174.2 S D=8.0 \mathrm{~cm}$; body mass mean $72.4, S D=11.3 \mathrm{~kg}$ ) with less than one year surfing experience and participated in the sport less than once per month.

Task 
The second experiment was carried out in a similar manner to that of Experiment 1, but because participants were novices, task requirements differed. That is, participants were required to attempt to surf a $0.7 \mathrm{~m}$ high white water wave over a distance of $100 \mathrm{~m}$. Participants were required to 'pop up' onto the surfboard (i.e., moving from a lying to standing position on the surfboard) and 'trim' the surfboard along the wave (i.e., controlling the surfboard whilst being pushed by the wave). After a briefing, all participants received a consistent 15 min surfing safety lesson to ensure participants' safety. Novice surfers had two familiarisation trials, consisting of two waves, prior to the start of the experiment.

\section{Procedure}

The experiment consisted of three phases: pre-test, acquisition and transfer. During the acquisition phase ( 8 trials), participants received attentional focus instructions between each trial, with a period of 3 mins between trials. As in Experiment 1, comparability between EFOA and IFOA was the aim when developing instructions and participants in each groups were provided with generic instructions designed to create an external or internal focus, respectively (see table 2.) The transfer phase consisted of a single trial and was performed without focus instructions.

As in to Experiment 1, participants were asked to rate their adopted respective attentional foci (whilst surfing) along a 10-point Likert scale. The scale assessed the extent to which they adopted a focus on their movements or any part of their body (e.g., legs, torso, arms, hands or head) to a focus on their board, the wave, or other markers (e.g., fence, noise). A strong movement-centred focus was reflected with a score closer to 1 and a strong movement-effect focus was reflected in a score closer to 10.

\section{Dependent Measures and Analyses}

Cognitive anxiety was manipulated and assessed in the same manner as Experiment 1.

Surfing performance was assessed by the percentage of successful wave riding as defined by controlled standing on the surfboard for a minimum of 3 seconds i.e., any wave surfed for a minimum of 3 seconds was classified as being 'successful'. Any wave where participants failed to 'pop-up' or 'popped up' but failed to maintain a standing position for at least 3 seconds was classed as 
'unsuccessful'. Thus, there was no additional performance benefit for surfing a wave for longer than the required 3 seconds. Similar to Experiment 1, surfing performance was assessed via separate independent samples t-tests. Specifically, we compared the percentage of successful wave riding between the EFOA and IFOA groups across the different experimental phases (pre-test; acquisition; transfer) in addition to calculating each group's performance change score between pre-test and transfer and again between acquisition and transfer and submitting means to separate independent samples t-test. As in Experiment 1, separate independent samples t-tests were conducted at pre-test, acquisition, and transfer to compare the MRF-3 group means of the selfreported cognitive anxiety (worry) scale data. Furthermore, within group change scores were calculated between acquisition and transfer performance and the means subsequently submitted to an independent samples t-test to compare groups.

\section{Results}

\section{FOA Manipulation Check}

An independent samples t-test comparing the mean data of the EFOA and IFOA groups revealed that participants in the EFOA group reported significantly higher values (mean $=6.45, S D=$ 1.75; values more representative of a movement-effect focus) compared to those in the IFOA group (mean $=3.08, S D=0.90$; values more representative of a movement-centred focus) $\left(\mathrm{t}_{(19)}=5.88, p\right.$ $<.001)$.

Performance

As shown in Figure 2A, analysis revealed that there were no significant differences in performance between the IFOA and EFOA groups at pre-test $(\mathrm{EFOA}$ mean $=22, S D=44$; IFOA mean $\left.=14, S D=36 ; \mathrm{t}_{21}=0.471, p=0.643\right)$. However, the EFOA performed significantly better than the IFOA group during both acquisition (EFOA mean $=53, S D=24$; IFOA mean $=26, S D=31 ; \mathrm{t}_{21}$ $=2.215, p<0.05)$ and transfer $\left(\right.$ EFOA mean $=77, S D=44 ;$ IFOA mean $=36, S D=49 ; \mathrm{t}_{21}=2.123, p<$ 0.05). Performance change from pre-test to transfer conditions approached significance between the groups ( $\left.\mathrm{t}_{21}=1.42, p=0.08\right)$ with performance increasing by $55 \%$ in EFOA and by $21 \%$ in the IFOA. 


\section{Performance change from acquisition to transfer were not significantly different between the}

$\operatorname{groups}\left(\mathrm{t}_{21}=-.77, p=.45\right)$

\section{Anxiety}

As shown in Figure 2B, analyses of the anxiety data revealed that the self-reported anxiety levels did not differ between groups at either pre-test $(\mathrm{EFOA}$ mean $=2.44, S D=1.02$; IFOA mean $=$ $\left.3.93, S D=2.17 ; \mathrm{t}_{21}=-1.914, p=0.069\right)$, acquisition $($ EFOA mean $=3.11, S D=1.27$; IFOA mean $=$ $\left.4.00, S D=2.29 ; \mathrm{t}_{21}=-1.060, p=0.301\right)$, or transfer $(\mathrm{EFOA}$ mean $=4.56, S D=2.204$; IFOA mean $=$ 4.93, $\left.S D=1.77 ; \mathrm{t}_{21}=-0.429, p=0.673\right)$.

\section{Discussion}

Experiment 2 was conducted to determine whether the findings of Experiment 1 would extend to the novice population. Results revealed that whilst the level of surfing was not different at the start of testing (pre-test), the EFOA group performed significantly better than the IFOA at both acquisition (when explicitly FOA instructions were present) and transfer (where pressure to perform was introduced and explicit FOA instructions were not present). As such, it appears that an EFOA is a powerful performance tool when performing under both acquisition and competition situations regardless of expertise.

\section{General Discussion}

\section{Summary}

The main objective of the current investigation was to examine whether the benefits associated with an EFOA could be extended to the performance of the complex task of surfing and pressured conditions. The adoption of an EFOA has been shown to have positive effects on learning and performance for a range of sports (for a review see, Wulf, 2013). However, these effects have not previously been explored in a study that simultaneously compares both high and low-pressured conditions and includes both novice and advanced samples. Therefore, Experiment 1 examined the effects of attentional focus on advanced surfing performance under low and high-pressure conditions in an ecologically valid setting. Experiment 2 investigated whether acquiring and practicing complex 
motor skill manoeuvres under the adoption of an EFOA relative to an IFOA, removed the negative effects on performance typically observed under conditions of pressure (i.e., choking) and promoted coping. The results of the present investigation support the positive effects of adopting an EFOA on surfing performance in both advanced and novice surfers under both normal and pressured conditions.

\section{Pressure Manipulation}

The effects of the pressure manipulation of both Experiment 1 and Experiment 2 revealed that whilst resultant anxiety levels did not differ between groups at any experimental phase, the levels reported were medium according to the self-report measure adopted. Furthermore, the levels of anxiety reported at the experimental phases where anxiety was not targeted (pre-test and acquisition) were higher than expected. We speculate that this is due to participants in both experiments performing all trials in direct sight of a public viewing window that looks out onto the surfing lagoon. This is likely to have acted as an ego threatening 'crowd style' pressure manipulation resulting in a feeling of monitoring apprehension and a subsequent increase in the reported levels of anxiety during the non-pressured trials (i.e., pre-test and acquisition). In light of this, readers should be explicitly aware that our pressured conditions refer to situations of medium anxiety and the normal pressured conditions involved performances where possible public viewing was present; likely resulting in a level of self-reported anxiety that is higher than would be typical in a true non-pressured condition. As such, the remainder of this general discussion focuses on performance benefits experienced when adopting an EFOA under medium anxiety conditions rather than explicitly discussing how an EFOA may prevent choking. Further rationale for this performance explanation approach centres on the findings that choking was not explicitly observed when 'regular' performance to transfer change scores were compared between FOA groups for either experiment 1 or 2. That is, whilst pre-test to transfer data revealed that the EFOA groups experienced significant increases in performance compared to IFOA groups, the same is not true once our experimental manipulation had occurred and performance had been influenced by the manipulated FOA.

\section{Performance Benefits associated with an EFOA}

The results of both experiments revealed that after receiving attentional focus instructions, 
surfing performance was significantly better in the EFOA compared to the IFOA group. With advanced surfers gaining a competitive advantage of 1.2 points and novices having approximately a $30 \%$ increase (53\%compared to $26 \%$ ) in successfully catching and riding a wave. The benefits of adopting an EFOA over an IFOA were also present in the transfer pressure tests of both experiments. Specifically, performance benefits here manifested in an advantage score of a 1.03 competition performance points, and approximately a $40 \%$ increase in successfully catching and riding waves ( $78 \%$ compared to $36 \%)$.

\section{Theoretical explanations for the benefits of an EFOA on the performance of novices under}

\section{medium levels of pressure}

The phenomenon of coping under pressure as a consequence of inducing an EFOA is comparable to previous research (Bell \& Hardy, 2009, Lawrence et al., 2012; Mullen et al., 2012). This has been accounted for with self-focus theories (e.g., Baumeister, 1984; Masters, 1992; Masters \& Maxwell, 2008) and distraction based theories (Eysenck \& Clavo, 1992; Eysenck et al., 2007). We propose that self-focus theories are more affiliated to an explanation of the novice data. For example, the self-focus centred proposals of Masters' (1992) reinvestment theory, or conscious processing hypothesis, indicate that pressure increases state anxiety and self-awareness about performing a skill successfully. This, in turn, causes performers to 'reinvest' in previously developed explicit rules about performing the skill in an attempt to control the mechanics of the movement, resulting in a reduction in performance (Masters \& Maxwell, 2004). The novices in the current study were in the cognitive stage of learning (Fitts and Posner, 1967) had not yet developed any 'rules' about how to perform the surfing task since it was novel. Furthermore, the FOA literature indicates that participants who practice under an EFOA adopt a more automatic control of action (for a review see Wulf 2013). Therefore, we speculate that this automatic control of action led to the novices in the EFOA group developing less of the typical declarative, self-focused, step-by step rules about how to perform the task in comparison to their IFOA counterparts. The relative inhibition in the accumulation of explicit, movement-centred information in the EFOA compared to the IFOA resulted in less opportunity for reinvestment and thus greater performance under both acquisition and transfer (both medium pressured conditions according to our self-report measures of anxiety) (also see Lawrence et al., 
2012). This explanation is similar to that of adopting implicit learning strategies in an attempt to maintain or enhance performance under pressured situations (Mullen et al., 2005). In line with OPTIMAL theory, we account for the performance benefits of an EFOA at both acquisition and transfer with the notion that adopting an EFOA enhanced goal-action coupling whilst simultaneously reducing self-focus and increasing goal directed attention (Wulf \& Lewthwaite, 2016). Thus, adopting an EFOA may have combated the 'typical' (or though not observed in the current experiments) decrements in performance associated with the presence of pressure, by shifting attention away from the self and towards the goal. This shift is likely to have contributed to our observed increase in task performance and increase in motor learning. According to the OPTIMAL theory, this enhanced performance will have generated increased performance expectations of participants: one of the primary factors proposed in the theory for the increased goal-action coupling, thus creating a cycle of enhanced learning in both our acquisition and transfer conditions when participants adopted an EFOA.

Theoretical explanations for the benefits of an EFOA on the performance of experts when under medium levels pressure

We propose that the adyanced sample data of the current study correspond with distraction theories (Eysenck \& Clavo, 1992; Eysenck et al., 2007). Here task-irrelevant cues, such as state anxiety, compete with task-relevant information for limited cognitive resources. Specifically, attentional control theory (Eysenck et al., 2007) proposes that cognitive anxiety occupies processing and storage space of working memory, leading to a decrease in available task resources and a subsequent decrease in performance. The advanced surfers (performers who have already accumulated explicit task rules during the cognitive and associated stages of learning where they invested numerous hours to become proficient at the skill) may have benefited from more available attentional capacity and thus been afforded relatively more resources to enhance performance (in comparison to those advanced surfers adopting an IFOA) under the medium levels of pressure reported in the acquisition and transfer conditions. In terms of the FOA literature, similar to our previous explanation for the novice sample, it is possible that the stronger goal-action coupling, 
established from the adoption of an EFOA, resulted in increased performance and subsequently enhanced performance expectations (relative to the IFOA group); perhaps via increased motivation, reduced perceptions of task difficulty, and/or positive affect (Wulf \& Lewthwaite, 2016). Furthermore, it is possible that adopting an EFOA reduced cognitive demands resulting in more available attentional capacity compared to adopting an IFOA (see OPTIMAL Theory;

Wulf \& Lewthwaite, 2016). Whilst the current study did not provide any direct measures of attentional load, the interpretation that adopting an EFOA results in greater attentional capacity relative to adopting an IFOA is based on the experimental data of Wulf and Colleagues (Wulf et al., 2001). Here researchers demonstrated that reaction times to a secondary attention-demanding task were significantly faster under an EFOA (compared to an IFOA) when performing a primary balancing task (balance performance was also superior under the EFOA condition). We acknowledge that the explanations in both this and the previous paragraph are speculative since the methodologies of Experiment 1 and 2 do not afford specific investigation into the proposed self-focus mechanisms of OPTIMAL theory and reinvestment theory or the distraction and shifting mechanisms within the ACT. It is plausible that all interacted to play a part in our findings, but disentangling the complexities behind these mechanisms requires further investigation.

\section{Conclusion}

In summary, the current investigation utilised a complex task in an ecologically valid (but controllable) setting to examine the effects of FOA on both advanced and novice surfing performance under medium levels of performance pressure. Whilst the effect of FOA on pressured performance has previously been investigated within novice (Lawrence et al., 2012) and expert populations (Bell \& Hardy, 2009), this has not been investigated in a single study or in an ecologically valid setting.

Current findings offer support for the existing FOA body of literature, with EFOA leading to greater performance (compared to an IFOA) for both novices and experts under both normal and competitive situations. As such, surfers of all levels (and those instructing surfing), could enhance performance in both training and competition by using of an EFOA (e.g., focusing on the movement of the board, wetsuit or clothing, wristwatch) and preventing the use of cues and instructions that focus on the body (e.g., the movement of the arms, legs, torso, head). Given that large sections of the International 
Surfing Association's coaching award manuals and resources utilise body centred (internally focused) coaching points, encouraging the adoption of an EFOA would likely require a shift in direction in both coaching 'paperwork' and coaching behaviour. Whilst changing the language of coaching points within existing coaching resources may sound like a huge task for any sport, the performance gains made significantly outweigh the effort. Furthermore, the changes needed do not require the description of new sport specific biomechanical techniques. Rather, the desired effect would be achieved by completing the relatively simple task of changing descriptive coaching language from body focused to effect/outcome focused e.g., changing the language from 'rotate the chest' to rotate the wetsuit logo' or from 'lean the body towards the face of the wave' to 'tip the rail of the board towards the face of the wave'.

\section{References}

Allsop, J., Lawrence, G. P., Gray, R., \& Khan, M.A. (2017). The interaction between practice and performance pressure on the planning and control of fast target directed movement. Psychological Research, 81(5): 1004-1019

Baumeister, R.F. (1984). Choking under pressure: Self-consciousness and paradoxical effects of incentives on skilful performance. Journal of Personality and Social Psychology, 46, 610-620. Beilock, S.L., \& Carr, T.H. (2001). On the fragility of skilled performance: What governs choking under pressure? Journal of Experimental Psychology: General, 130, 701-725.

Beilock, S.L., Carr, T.H., MacMahon, C., \& Starkes, J. (2002). When paying attention becomes counterproductive: Impact of divided versus skill-focused attention on novice and experienced performance of sensorimotor skills. Journal Experimental Psychology: Applied, $8,6-16$

Bell, J.J., \& Hardy. J. (2009). Effects of attentional focus on skilled performance in golf. Journal Applied Sport Psychology, 21, 163-177.

Cassell, V., Beattie, S., \& Lawrence, G.P. (2017). Changing performance pressure between training and completion influences action planning because of reduction in the efficiency of action execution. Anxiety, Stress, \& Coping, 31(1): 1-14 
Castaneda, B., \& Gray, R. (2007). Effects of focus of attention on baseball batting performance in players of differing skill levels. Journal of Sport and Exercise Psychology, 29, 60-77.

Collins, D., Carson, H.J., \& Toner, J. (2016). Letter to the editor concerning the article "Performance of gymnastics skill benefits from an external focus of attention" by Abdollahipour, Wulf, Psotta \& Nieto (2015). Journal of Sports Sciences, 34, 1288-1292.

DeCaro, M.S., Thomas, R.D, Albert, N.B., \& Beilock, S.L. (2011). Choking under pressure: Multiple routes to skill failure. Journal of Experimental Psychology: General, 140, 390-406.

Eysenck, M.W., \& Calvo, M.G. (1992). Anxiety and performance: The processing efficiency theory. Cognition and Emotion, 6, $409-434$.

Eysenck, M.W., Derakshan, N., Santos, R., \& Calvo. M.G. (2007). Anxiety and cognitive performance: Attentional control theory. Emotion, , , 336-353.

Fitts, P.M., \& Posner, M.I. (1967). Human performance. Oxford, England: Brooks/Cole.

Ford, P., Hodges, N.J., \& Williams, A.M. (2005). Online attentional-focus manipulations in a soccer dribbling task: Implications for the proceduralization of motor skills. Journal of Motor Behavior, 37, 386-394. McMahon

Guadagnoli, M.A., \& Lee, T.D. (2004). Challenge point: A framework for conceptualizing the effects of various practice conditions in motor learning. Journal of Motor Behavior, 36, 212-224.

Gucciardi, D. F., Longbottom, J. L, Jackson, B., \& Dimmock, J. A. (2010). Experienced golfers' perspectives on choking under pressure. Journal of Sport \& Exercise Psychology, 32(1), 6183.

Hosp, J.A., Pekanovic, A., Rioult-Pedotti, M.S., \& Luft, A.R. (2011). Dopaminergic Projections from Midbrain to Primary Motor Cortex Mediate Motor Skill Learning. Journal of Neuroscience, $31,2481-2487$.

International Surfing Association (2016).

Krane, V. (1994). The Mental Readiness Form as a measure of competitive state anxiety. The Sport Psychologist, 8, 189-202. 
Lawrence, G.P., Cassell, V., Beattie, S., Woodman, T., Gottwald, V.M., Khan, M.A., \& Hardy, L. (2014). Practice with anxiety improves performance, but only when anxious: Evidence for the specificity of practice hypothesis. Psychological Research, 78, 634-650.

Lawrence, G.P., Gottwald, V.M., Khan, M.A., \& Kramer, R.S (2012). The movement kinematics and learning strategies associated with adopting different foci of attention during both acquisition and anxious performance. Frontiers in Movement Science and Sport Psychology, 3, 468-475.

Masters, R.S.W. (1992). Knowledge, knerves and know-how. British Journal of Psychology, 83, 343358.

Masters, R., \& Maxwell, J. (2008). The theory of reinvestment. International Review of Sport and Exercise Psychology, 1, 160-183.

Mullen, R., Hardy, L., \& Tattersall, A. (2005). The effects of anxiety on motor performance: A test of the conscious processing hypothesis. Journal of Sport and Exercise Psychology, 27, 212-225.

Mullen, R., Faull, A., Jones, E.S., \& Kingston, K. (2012). Attentional focus and performance anxiety: effects on simulated race-driving performance and heart rate variability. Frontiers of Psychology, 3, 426.

Oudejans, R. R. D., \& Pijpers, J. R. R. (2010). Training with mild anxiety may prevent choking under higher levels of anxiety, Psychology of Sport and Exercise, 11(1), 44-50.

Prinz, W. (1997). Perception and action planning. European Journal of Cognitive Psychology, 9, 129154.

Robazza, C., Bortoli, L., \& Nougier, V. (2000). Performance emotions in an elite archer: A case study. Journal of Sport Behavior, 23, 144-163.

Totsika, V., \& Wulf, G. (2003). The influence of external and internal foci of attention on transfer to novel situations and skills. Research Quarterly in Exercise and Sport, 74, 220-225.

Wilson, M.R., Wood, G., \& Vine, S.J. (2009). Anxiety, attentional control, and performance impairment in penalty kicks. Journal of Sport and Exercise Psychology, 31, 761-775.

Wulf, G. Attentional focus and motor learning: a review of 15 years. (2013). International Review of Sport and Exercise Psychology, 6, 77-104. 
Wulf, G., Höß, M., Prinz, W. (1998). Instructions for motor learning: Differential effects of internal versus external focus of attention. Journal of Motor Behavior, 30, 169-179.

Wulf, G., \& Lewthwaite, R. (2010). Effortless motor learning? An external focus of attention enhances movement effectiveness and efficiency. In B. Bruya (Ed.), Effortless Attention: A New Perspective in Attention and Action (pp. 75-101). Cambridge: MIT Press.

Wulf, G., \& Lewthwaite, R. (2016). Optimizing performance through intrinsic motivation and attention for learning: The OPTIMAL theory of motor learning. Psychonomic Bulletin Review, 23, 1382-1414.

Wulf, G., McConnel, N., \& Gärtner, M. (2002). Enhancing the learning of sport skills through external-focus feedback. Journal of Motor Behavior, 34, 171-182.

Wulf, G., \& McNevin, N.H. (2003). Simply distracting learners is not enough: More evidence for the learning benefits of an external focus of attention. European Journal of Sport Science, 3, 113.

Wulf, G., McNevin, N.H., \& Shea, C.H. (2001). The automaticity of complex motor skill learning as a function of attentional focus. Quarterly Journal of Experimental Psychology, 54A, 11431154

Wulf, G., \& Prinz, W. (2001). Directing attention to movement effects enhances learning: A review. Psychonomic Bulletin \& Review, 8, 648-660. 
Table 1. Attentional Focus Instruction Guidelines for Advanced Surfers

\begin{tabular}{|c|c|c|}
\hline Skill/task & IFOA Instructions & EFOA Instructions \\
\hline Bend more & Bend knees & Compress \\
\hline Stand taller & Straighten legs & Extend \\
\hline $\begin{array}{l}\text { Ensure dynamic position } \\
\text { during bottom turn }\end{array}$ & $\begin{array}{l}\text { Rotate upper body during } \\
\text { bottom turn }\end{array}$ & $\begin{array}{l}\text { Move 'wristband' to top } \\
\text { wave }\end{array}$ \\
\hline $\begin{array}{l}\text { Ensure dynamic position } \\
\text { during top turn }\end{array}$ & $\begin{array}{l}\text { Throw back arm over head } \\
\text { during top turn }\end{array}$ & $\begin{array}{l}\text { Throw 'wristband' over the } \\
\text { nose (front of the board) } \\
\text { during top turn }\end{array}$ \\
\hline Increase speed & More weight on front foot & $\begin{array}{l}\text { More weight on nose (front of } \\
\text { the board) }\end{array}$ \\
\hline Reduce speed & $\begin{array}{l}\text { More weight on back/front } \\
\text { foot }\end{array}$ & $\begin{array}{l}\text { More weight on tail (back of } \\
\text { the board) }\end{array}$ \\
\hline $\begin{array}{l}\text { Lead into turns with } \\
\text { dynamic position }\end{array}$ & $\begin{array}{l}\text { Turn head in specific } \\
\text { direction }\end{array}$ & $\begin{array}{l}\text { Focus on specific section of } \\
\text { the wave }\end{array}$ \\
\hline
\end{tabular}

Table 2. Attentional Focus Instructions for Novice Surfers

\begin{tabular}{lll}
\hline Skill/task & EFOA Instructions \\
Bend more & Compress \\
Stand taller & Extend \\
$\begin{array}{l}\text { Ensuring a good position on } \\
\text { popup }\end{array}$ & Lift head up during 'popup' & $\begin{array}{l}\text { Focus on point in distance } \\
\text { during 'popup' }\end{array}$ \\
$\begin{array}{l}\text { Eurn the board } \\
\text { when starting a turn }\end{array}$ & $\begin{array}{l}\text { Point your fingers to right or } \\
\text { left }\end{array}$ & $\begin{array}{l}\text { Move your 'wristband' to the } \\
\text { right or left }\end{array}$ \\
$\begin{array}{l}\text { Increase speed to prevent } \\
\text { falling off the back of the } \\
\text { wave }\end{array}$ & More weight on front foot & $\begin{array}{l}\text { More weight on nose (front of } \\
\text { the board) }\end{array}$ \\
\hline
\end{tabular}


A.

-EFOA Advanced

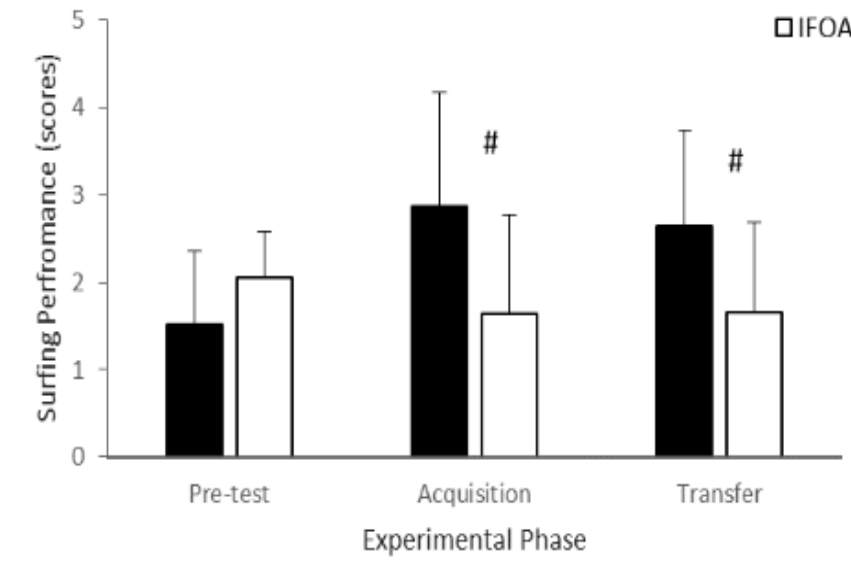

B.

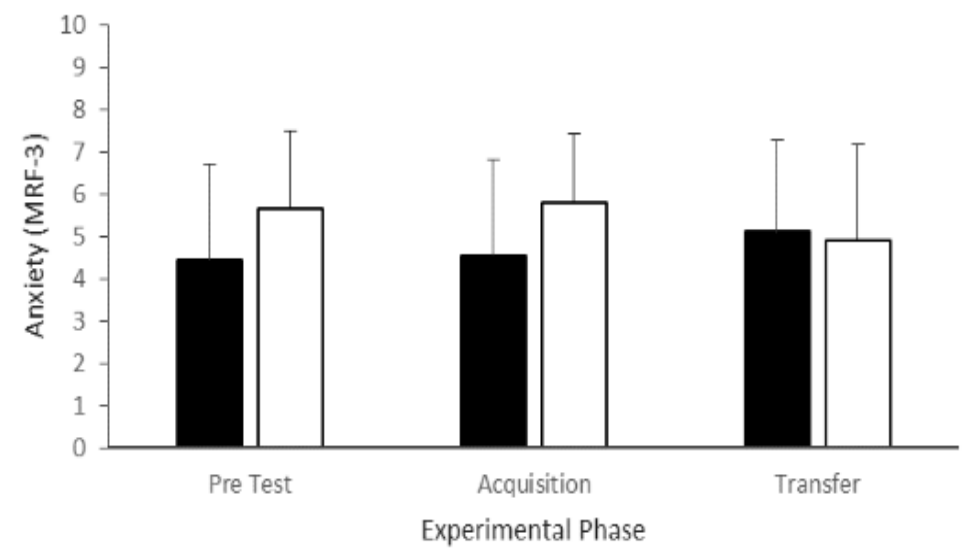

Figure 1. Performance (A) and anxiety (B) scores of advanced surfers as a function of group (EFOA = external focus of attention; IFOA = internal focus of attention) and experimental phase. \# Signifies a difference between focus of attention groups at the time point indicated $(P<0.05)$. 
A.

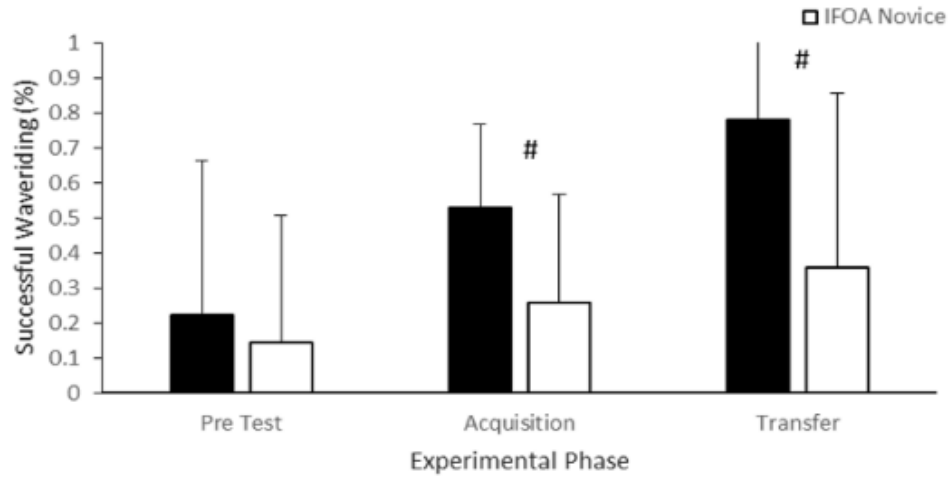

B.

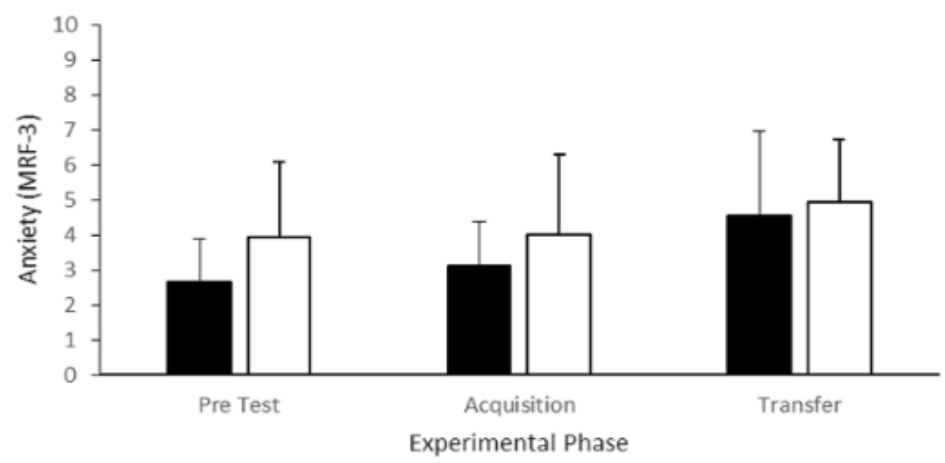

Figure 2. Percentage of successful waveriding (A) and anxiety (B) scores of novice surfers as a function of group (EFOA = external focus of attention, IFOA = internal focus of attention) and experimental phase. \# Signifies a difference between focus of attention groups at the time point indicated $(P<0.05)$. 